\title{
TV/Series
}

$14 \mid 2018$

Posthumains en séries

\section{Introduction : Posthumains en séries}

Hélène Machinal et Monica Michlin

\section{(2) OpenEdition}

Journals

Édition électronique

URL : http://journals.openedition.org/tvseries/3349

DOI : 10.4000/tvseries.3349

ISSN : 2266-0909

Éditeur

GRIC - Groupe de recherche Identités et Cultures

Référence électronique

Hélène Machinal et Monica Michlin, «Introduction : Posthumains en séries », TV/Series [En ligne], 14 | 2018, mis en ligne le 31 décembre 2018, consulté le 24 septembre 2020. URL : http:// journals.openedition.org/tvseries/3349; DOI : https://doi.org/10.4000/tvseries.3349

Ce document a été généré automatiquement le 24 septembre 2020.

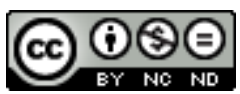

TV/Series est mis à disposition selon les termes de la licence Creative Commons Attribution - Pas d'Utilisation Commerciale - Pas de Modification 4.0 International. 


\title{
Introduction : Posthumains en séries
}

\author{
Hélène Machinal et Monica Michlin
}

1 Posthumains en séries poursuit une réflexion critique qui a connu une accélération depuis le début $\mathrm{du} \mathrm{xxI}^{\mathrm{e}}$ siècle sur le posthumain et sur la redéfinition de l'identité humaine que ce concept problématise. Les articles réunis ici croisent les approches de spécialistes des cultures américaine et britannique, de comparatistes et de spécialistes de cinéma et d'arts visuels. La recherche sur le posthumain, en France, reste très ancrée dans le champ de la philosophie, avec les ouvrages de Dominique Lecourt (Humain, posthumain, 2003), Yves Michaud (Humain, inhumain, trop humain, 2006), ou Thierry Hoquet (Cyborg philosophie, 2011). Dans la recherche anglophone, l'approche philosophique occupe aussi, à l'ère contemporaine, une place de choix, de N. Katherine Hayles (How We Became Posthuman, 1999) à Cary Wolfe (What is Posthumanism?, 2010). Hayles, tout comme Donna Haraway (Cyborg Manifesto, 1984) ou Rosi Braidotti (The Posthuman, 2013) ${ }^{1}$, est une philosophe féministe revendiquant une approche postcoloniale, ce qui pointe d'emblée la dimension politique (et souvent intersectionnelle) à l'œuvre dans ces théorisations d'une posthumanité. Les chercheuses et chercheurs qui analysent la figuration du posthumain dans la fiction se retrouvent donc à la croisée de plusieurs champs disciplinaires, et ont en commun un intérêt pour l'imaginaire de la science que la fiction déploie pour projeter futurs possibles et devenirs de notre espèce. Les ouvrages actuels sont nombreux, et le posthumain se décline souvent à partir du dualisme cartésien entre corps augmentés par la (bio)technologie ou esprits modifiés par l'informatique et le numérique, comme dans Le Sujet digital (2015)², réunissant des chercheurs de différentes disciplines de l'Université Paris 8 Vincennes Saint-Denis.

2 Rares, cependant, sont les analyses consacrées uniquement aux séries télévisées. Un ouvrage comme celui de Daniel Dinello, Technophobia! Science Fiction Visions of Posthuman Technology $(2005)^{3}$ étudie le posthumain sous toutes ses facettes, de la cybernétique, au cyborg, aux bio- et nano-technologies et aux virus (affectant tant le corps humain que la machine), mais son corpus reste principalement cinématographique. En France, la 
fiction qui traite des déclinaisons du posthumain est au cœur de deux ouvrages issus de colloques s'étant tenu à Brest et à l'UQAM sous l'impulsion d'Elaine Després, Hélène Machinal et Jean-François Chassay - PostHumains. Frontières, évolutions, hybridités (2014) ${ }^{4}$, et Les Frontières de l'humain et le posthumain $(2014)^{5}$ - puis, dans le sillage de ces deux volumes, Mutations I : corps posthumains ${ }^{6}$. Parmi les opus récents comprenant des articles sur la fiction télévisée sérielle - et en particulier sur les séries Black Mirror et Westworld, qui font également l'objet de microlectures dans ce numéro de TV/Series - citons Hybridités posthumaines: cyborgs, mutant/e/s, hackers (2018) ${ }^{7}$, et Mutations II : homme/ machine (2018) ${ }^{8}$.

Plus spécifiquement sur les séries, Otrante a récemment proposé un numéro consacré aux séries de genre, mais les articles portaient sur le fantastique et la science fiction plus largement, sans se restreindre aux figures du posthumain. Après le numéro 11 de $T V /$ Series consacré à la série Battlestar Galactica ${ }^{9}$ - qui analysait (notamment dans le reboot de Ronald D. Moore et dans son prequel Caprica ${ }^{10}$ ), les différences entre humain et posthumain, la remise en question du dualisme cartésien ${ }^{11}$, le rêve posthumaniste voire transhumaniste ${ }^{12}$, le robot souffrant comme un être humain en devenir ou «en souffrance ${ }^{13}$, les représentations à l'écran de l'être machinique par des acteurs et actrices «organiques ${ }^{14}$ - ce numéro consacré au posthumain sériel se propose donc d'apporter une pierre aux représentations du posthumain dans les séries télévisées contemporaines.

4 Le posthumain peut se décliner selon les classifications proposées par Thierry Hoquet ${ }^{15}$, du robot au clone, au zombie ou au virus, mais nous avons ici privilégié des séries qui mettent en scène des confrontations, hybridations, augmentations et déclinaisons entre l'être humain et la machine. Depuis Do Androids Dream of Electric Sheep (Philip K. Dick, 1966) et sa version filmique Blade Runner (Ridley Scott, 1982) puis Blade Runner 2049 (Denis Villeneuve, 2017), l'opposition entre des êtres humains capables de ressentir des émotions et des êtres machiniques qui en seraient dépourvus apparaît comme une dichotomie simpliste. En effet, les entités machiniques ont beaucoup évolué depuis les débuts de la robotique, et de nombreuses séries mettent en fiction des machines qui semblent plus humaines que les femmes et les hommes qui les entourent: dans ces mondes diégétiques, le robot est devenu humanoïde, et il n'est plus nécessaire d'avoir recours à un test de Turing pour déterminer si le non-humain ressent des émotions. Les êtres machiniques sont devenus l'enjeu de problématiques éthiques et politiques à mesure que la possibilité qu'elles deviennent sentientes et conscientes fait craindre des dangers opposés.

D'une part, celui que nous asservissions des autres qui seraient nos semblables. Depuis le tournant numérique du xxi ${ }^{\mathrm{e}}$ siècle, en effet, un nombre croissant de séries télévisées - celles de Ronald D. Moore citées plus haut, mais aussi Real Humans (Lars Lundström, SVT1, 2012-2014) et son remake américain Humans (AMC, 2015-), Dollhouse (Joss Whedon, Fox, 2009-2010), et plus récemment Westworld de Lisa Joy et Jonathan Nolan (HBO, 2016- ) - ont mis en scène des robots ayant atteint le stade de l'intelligence artificielle et ayant une apparence parfaitement humaine. Toutes les problématiques de l'esclavage se rejouent alors: car comment penser «machine» ce qui nous est semblable ? L'amour humain-machine devient-il possible ${ }^{16}$ ? À l'inverse, d'autres récits reflètent la peur que nous ayons créé des créatures non dénuées d'affects mais infiniment plus puissantes que nous, qui nous manipuleront et nous détruiront (retour du mythe de Prométhée et de ses nombreux avatars depuis Frankenstein $\left.{ }^{17}\right)$. Ces 
diégèses projettent nos craintes que les machines, pensant en réseau et à une rapidité inégalable par la pensée humaine, puissent « détrôner » le sujet humain, et le réduire en esclavage à son tour ${ }^{18}$.

6 Depuis le tournant numérique, face à la possibilité désormais proche que les IA prolifèrent, la frontière entre ce qui était fiction hier et sera réalité demain est devenu un topos de la fiction (souvent dystopique) comme du discours publicitaire (l'IA devenue le sésame et le totem de toute publicité pour appareils électroniques ${ }^{19}$ ). Désir d'immortalité numérique et malaise face à ce que serait un sujet constitué par la somme de données numériques téléchargées dans un corps de métal et de silicone se conjuguent ${ }^{20}$. Les séries étudiées dans ce volume - notamment Westworld et Black Mirror - proposent de manière hyper-réflexive des récits spéculatifs sur la redéfinition de l'humain que toutes ces évolutions technologiques pourraient entraîner dans un futur proche.

7 L'on argumentera ici que le temps long des séries audiovisuelles permet, mieux que le cinéma, de se représenter les lents processus de programmation, d'apprentissage et d'imitation ou de performance répétées par lesquels la machine devient humaine tandis que simultanément, l'humain se fait «hybride» par son interconnexion prolongée avec les machines, telle Root dans Person of Interest (Jonathan Nolan, ABC, 2011-2016). Le posthumain est la «matrice» idéale d'une narration à la fois «feuilletonnante » et épisodique, à l'instar de Dollhouse où chaque personnage joue un nouveau rôle par épisode après avoir été « reprogrammé » à cet effet. Le point de départ de Westworld est un programme (une fiction mise en abyme) que les androïdes répètent en boucle à l'infini et dont la série va pouvoir révéler, niveau par niveau (à l'image de la Mesa ou tour de contrôle du parc où se déroule l'action) les couches, les coulisses et le déraillement... Même dans l'anthologie Black Mirror, où l'on ne suit pas de semaine en semaine la progression d'une même diégèse, l'accumulation de représentations dystopiques d'un futur proche permet de décliner le posthumain dans notre rapport aux écrans et au virtuel, représentés via une interface écranique dans une mise en abyme qui crée délibérément le malaise.

8 Si l'on assiste, dans ces récits sériels, à la remise en question de la souveraineté et de la supériorité du sujet humain dans sa « mise en relation » à la fois informatique, affective et physique avec l'autre machinique (virtuel ou posthumain), on peut se demander si le double but - divertissement et réflexion éthique/politique - de ces séries n'est pas en soi contradictoire. Que penser de la mise en abyme du divertissement et de la notion "d'immersion» sensorielle dans une série comme Westworld? Peut-on véritablement penser jusqu'au bout la mise en abyme de la surveillance dans des séries comme Black Mirror ou Person of Interest, ou ces séries reposent-elles au contraire sur notre déni du monde dans lequel nous vivons déjà ? Comment penser la réflexivité que ces séries à narration complexe nous proposent ? La mise en abyme de la posture spectatorielle estelle éthiquement cohérente dans Westworld? Tels sont quelques uns des enjeux que ce numéro de TV/Series entend aborder à travers une sélection d'articles issus de deux journées d'études organisées, l'une sur Westworld à l'Université de Bretagne Occidentale à Brest en septembre 2017, et l'autre en novembre 2017 à l'Université Paul-Valéry Montpellier 3, sur le posthumain dans les séries télévisées.

9 Une première série d'articles se penche sur l'intertexte shakespearien des séries mettant en scène des posthumains. Dans " "What a piece of work is your machine, Harold": Shakespeare et la réinvention de l'humanité dans les séries américaines 
d'anticipation ", Sarah Hatchuel nous propose de remonter aux sources de la culture populaire en se penchant sur les références à Shakespeare dans les séries mettant en scène des créatures posthumaines. Au-delà de la dimension intertextuelle, les citations de l'auteur élisabéthain dans la bouche des posthumains serait le signe de leur humanisation, hypothèse dont la récurrence s'illustre en fiction sérielle dans Star Trek ou Person of Interest avant Westworld. Le dépassement des oppositions entre identité et altérité, humain et non-humain passerait par un détour shakespearien déclencheur d'une «extension du domaine de l'humain». Toujours à partir d'une définition de l'humain s'appuyant sur la capacité à l'empathie dans une œuvre de Shakespeare, Delphine Lemonnier-Texier convoque dans « Posthumain et réception, de The Tempest à Westworld», le phénomène de "la vallée de l'étrange " théorisé par le roboticien Masahiro Mori pour montrer que la série Westworld fait entrer ses spectatrices et spectateurs dans un jeu de mise en abyme qui construit une réception spécifique des créatures posthumaines. La contribution de Delphine Lemonnier-Texier et Sandrine Oriez " "It wasn't a dream, [...] it was a memory" : Le fonctionnement de quelques intertextes dans Westworld» élargit encore l'étude de la richesse intertextuelle de la série et revient sur la "double maïeutique " qui en découle pour les spectatrices et spectateurs mais aussi pour les personnages. À nouveau, c'est la réfraction entre humains et nonhumains qui permet une exploration de l'humanité de l'être.

Gaïd Girard ${ }^{21}$, dans "Retour vers la matrice: le Westworld (Mondwest) de Michael Crichton (1973)», nous fait remonter aux origines de la série Westworld, et nous rappelle que la série fut d'abord un film, resté célèbre pour le rôle du robot androïde joué par Yul Brynner. Contrairement au film, qui instaurait une distance ironique par rapport aux codes de la masculinité, à ceux du genre et de la société de consommation, la série pratique cette distanciation par une dimension réflexive qui permet de problématiser des enjeux plus contemporains, comme l'argumente Florent Favard dans " "The maze wasn't made for you": Artificial consciousness and reflexive narration in Westworld (HBO, 2016-) ». Sa lecture s'attache à la question de l'accès à la conscience d'une entité machinique, qu'elle soit (presque) totalement désincarnée comme dans Person of Interest ou, qu'elle soit au contraire (presque) plus vraie que nature comme les personnages de Maeve et Dolores dans Westworld. Briac Picart-Hellec poursuit cette analyse de la réflexivité de la série Westworld en s'attardant, dans " "My own fucking story" : le raga de Westworld", sur le rôle de l'improvisation dans l'émancipation des machines ; pour devenir effective, celle-ci supposerait, in fine, une "disparition " des spectateurs et la fin de tout spectacle. Toujours sur le versant de la réception, Sébastien Lefait, qui signe " "Have you ever questioned the nature of your reality?" " Westworld (HBO, 2016-) et la culture du complot", souligne que la série a recours au récit complotiste pour créer un continuum transmedia hyper-réflexif.

Dans la suite de ce que le visionnage met (ou remet) en jeu sur le plan des rapports de pouvoir, Elisabeth Mullen s'intéresse à la série sous l'angle du genre, au double sens de " genre » et " gender ». Dans " "Not much of a rind on you": (De)Constructing Genre and Gender in Westworld (Lisa Joy and Jonathan Nolan, HBO, 2016-) ), elle montre que la série passe par un brouillage des références génériques pour explorer des enjeux liés à l'hégémonie masculine et à l'agentivité (agency) féminine. Benjamin Campion poursuit cette réflexion sur le genre et sur les codes visuels et idéologiques de la série, dans « Le sexe sans le sexe: convergence de la nudité frontale et de l'empowerment féminin dans Westworld". 

Mirror, et se concentrent sur une facette spécifique du posthumain: la place de l'humain en culture de l'écran ${ }^{22}$. Après les explorations d'une altérité incarnée par des corps augmentés et/ou modifiés par la technologie, c'est davantage la société écranique elle-même ${ }^{23}$ et les artéfacts qui brouillent la frontière entre l'actuel et le virtuel ${ }^{24}$ que Black Mirror nous engage à considérer. David Roche creuse la question de la mémoire et de son devenir, "à l'ère du numérique, de la surveillance et de la culture de la convergence", signant "Mémoire et média dans "The Entire History of You" (Black Mirror S01E03) ${ }^{25} \%$. Hélène Machinal s'attache ensuite aux interactions entre réel et virtuel et à l'image matrice d'une fabrique ou d'un stockage du vivant dans «L'épisode "San Junipero" de Black Mirror: les miroitements du post-humain ». Pour clore cette séquence consacrée à la série de Charlie Brooker, Marie Baudoin, dans « Le dialogue des consciences : des interfaces posthumaines dans "Be Right Back" (Black Mirror S02E01) » analyse cet épisode en termes d'un test de Turing inversé, qui interrogerait ce qui est véritablement singulièrement « soi » dans la subjectivité humaine.

Pour conclure (temporairement) ce volume, déjà appelé à connaître une suite, Julien Achemchame propose une lecture hyper-métafilmique de la série Dollhouse de Joss Whedon (FOX, 2009-2010). Dans «Écho, un "corps-marchandise" posthumain au service de la sérialité audiovisuelle ", il démontre que la posthumaine Écho lutte pour son émancipation au sein d'un système qui, de par la réflexivité et l'intertextualité déployées dans la série, possède de nombreux points communs avec l'industrie hollywoodienne $\mathrm{du}$ divertissement. Série hybride articulant épisodique et feuilletonnant pour mieux multiplier les «rôles" de sa poupée vedette, Dollhouse sert de "véhicule » à son interprète principale, Eliza Dushku, tout en tendant un miroir sombre au star-system hollywoodien.

Ainsi, l'ensemble de ce numéro sur le posthumain dans les séries contemporaines aborde tant les questions de production (adaptation et ré-adaptation, remake sériel, " machine » hollywoodienne et "production en série »), que de réception: intertextes littéraires, filmiques, artistiques, et télévisuels convoqués explicitement par les séries elles-mêmes ou allusions plus discrètes à « décoder » par des spectateurs-herméneutes. La question de la performance de l'identité posthumaine dans la diégèse (simulacre, simulation, mise en abyme de la performance de l'acteur ou de l'actrice, construction performative "réitérée » de manière sérielle) occupe une place importante dans toutes les contributions, tout comme celle de l'interface écranique (téléviseur, ordinateur, tablette, smartphone) au cœur du visionnage de ces fictions qui interrogent notre propre statut de spectatrices et spectateurs hyperconnectés... et peut-être déjà un peu posthumains. 


\section{BIBLIOGRAPHIE}

ACHоUсHE Mehdi, «Caprica, l'utopisme technologique et le "cyborg spirituel” ", TV/Series n¹1, 2017, mis en ligne le 11 juin 2017, http://journals.openedition.org/tvseries/2042, consulté le 29 décembre 2018.

BOOF-VERMESSE Isabelle, Jean-François Chassay (éd.), Mutations II : homme/machine, Otrante $\mathrm{n}^{\circ} 43$, Paris, Kimé, 2018.

BOOF-VERMESSE Isabelle, Matthieu Freyheit, Hélène Machinal (éd.), Hybridités posthumaines : cyborgs, mutant/e/s, hackers, Orizons, 2018.

BRAIDOTTI Rosi, The Posthuman, Cambridge et Malden (MA), Polity Press, 2013.

CHASSAY Jean-François, Hélène Machinal (éd.), Mutations I : Corps posthumains, Otrante $\mathrm{n}^{\circ} 38$, Paris, Kimé, 2015.

DESPRÉS Elaine, Hélène Machinal (éd.), PostHumains. Frontières, évolutions, hybridités, Rennes, PUR, 2014.

DINELLo Daniel, Technophobia! Science Fiction Visions of Posthuman Technology, Austin, University of Texas Press, 2005.

GERVAIS Bertrand, Samuel Archibald, Sylvain David, Joanne Lalonde, Vincent Lavoie, Sylvano Santini (éd.), Soif de réalité, Montréal, Nota Bene, 2018.

HARAWAY Donna, «A Cyborg Manifesto: Science, Technology and Socialist-Feminism in the Late Twentieth Century » [1984], in Simians, Cyborgs and Women: The Reinvention of Nature, New York, Routledge, 1991, p. 149-181.

HAYLES N. Katherine, How We Became Posthuman: Virtual Bodies in Cybernetics, Literature and Informatics, Chicago, University of Chicago Press, 1999.

HOQUET Thierry, «Cyborg, Mutant, Robot, etc. Essai de typologie des presque-humains », in Posthumains. Frontières, évolutions, hybridités, éd. Elaine Després et Hélène Machinal, Rennes, PUR, 2014, p. 99-118.

-----, cyborg Philosophie, Paris, Le Seuil, 2011.

JENKINS Henry, Convergence Culture: Where Old and New Media Collide, New York, New York University Press, 2006.

KURZWEIL Raymond, The Singularity is Near: When Humans Transcend Biology, New York, Viking, 2005. LARSONNEUR Claire, Arnaud Regnauld, Pierre Cassou-Noguès, Sara Touiza (éd.), Le Sujet digital, Dijon, Presses du réel, 2015.

LECOURT Dominique, Humain, posthumain, Paris, PUF, 2014 [2003].

LEMIEUX René, « La machine comme "prothèse d'origine" : réflexion philosophique sur le sujet humain dans Battlestar Galactica de Ronald D. Moore ", TV/Series n¹1, 2017, mis en ligne le 11 juin 2017, https://journals.openedition.org/tvseries/1981, consulté le 29 décembre 2018.

LÉVY Ophir, « Le cylon est-il un humain en souffrance ? », TV/Series n¹1, 2017, mis en ligne le 11 juin 2017, http://journals.openedition.org/tvseries/2057, consulté le 29 décembre 2018. 
LIPOVETSKY Gilles, Jean Serroy, L'écran global : culture-médias et cinéma à l'âge hypermoderne. Paris, Éd. Le Seuil, coll. La couleur des idées, 2007.

MACHINAL Hélène, «Corps technologiques et environnements connectés : “The dynamic, fluid nature of reality" ou le corps face à l'écran ", TV/Series n¹1, 2017, mis en ligne le 11 juin 2017, https://journals.openedition.org/tvseries/2103, consulté le 29 décembre 2018.

MICHLIN Monica, « Introduction », Philosopher avec Battlestar Galactica, TV/Series n¹1, juillet 2017, https://journals.openedition.org/tvseries/2094, consulté le 29 décembre 2018.

TREMBLAY-CLÉROUX Marie-Ève, Jean-François Chassay (éd.), Les Frontières de l'humain et le posthumain, Cahiers Figura n³7, Presses Universitaires du Québec, 2014.

VITALI-ROSATI Marcello, Corps et virtuel : itinéraires à partir de Merleau-Ponty, Paris, L'Harmattan, 2010.

WOLFE Cary, What is Posthumanism?, Minneapolis and London, University of Minnesota Press, 2010.

\section{NOTES}

1. Rosi Braidotti, The Posthuman, Cambridge et Malden (MA), Polity Press, 2013.

2. Claire Larsonneur, Arnaud Regnauld, Pierre Cassou-Noguès et Sara Touiza (éd.), Le Sujet digital, Dijon et Paris, Presses du réel, 2015.

3. Daniel Dinello, Technophobia! Science Fiction Visions of Posthuman Technology, Austin, University of Texas Press, 2005.

4. Elaine Després et Hélène Machinal (éd.), PostHumains. Frontières, évolutions, hybridités, Rennes, PUR, 2014.

5. Marie-Ève Tremblay-Cléroux et Jean-François Chassay (éd.), Les Frontières de l'humain et le posthumain, Cahiers Figura n³7, Presses Universitaires du Québec, 2014.

6. Jean-François Chassay et Hélène Machinal (éd.), Mutations I: corps posthumains, Otrante $\mathrm{n}^{\circ} 38$, Paris, Kimé, 2015.

7. Isabelle Boof-Vermesse, Matthieu Freyheit et Hélène Machinal (éd.), Hybridités posthumaines: cyborgs, mutant/e/s, hackers, Orizons, 2018.

8. Isabelle Boof-Vermesse et Jean-François Chassay (éd.), Mutations II : homme/machine, éd. Otrante $\mathrm{n}^{\circ}$ 43, Paris, Kimé, 2018.

9. Battlestar Galactica (Greg Larson, ABC, 1978-1979) et Battlestar Galactica (Ronald D. Moore, Syfy, 2003-2009).

10. Caprica (Ronald D. Moore et Rémi Aubuchon, Syfy, 2010).

11. René Lemieux, «La machine comme "prothèse d'origine" : réflexion philosophique sur le sujet humain dans Battlestar Galactica de Ronald D. Moore ", TV/Series n¹1, 2017, mis en ligne le 11 juin 2017, https://journals.openedition.org/tvseries/1981, consulté le 29 décembre 2018.

12. Mehdi Achouche, "Caprica, l'utopisme technologique et le "cyborg spirituel" ", TV/Series $n^{\circ} 11,2017$, mis en ligne le 11 juin 2017, http://journals.openedition.org/tvseries/2042, consulté le 29 décembre 2018.

13. Ophir Lévy, «Le cylon est-il un humain en souffrance ? ", TV/Series $n^{\circ} 11,2017$, mis en ligne le 11 juin 2017, http://journals.openedition.org/tvseries/2057, consulté le 29 décembre 2018.

14. Hélène Machinal, «Corps technologiques et environnements connectés : "The dynamic, fluid nature of reality" ou le corps face à l'écran ", TV/Series n¹1, 2017, mis en ligne le 11 juin 2017, https://journals.openedition.org/tvseries/2103, consulté le 29 décembre 2018. 
15. Thierry Hoquet, «Cyborg, Mutant, Robot, etc. Essai de typologie des presque-humains », in Posthumains. Frontières, évolutions, hybridités, Elaine Després et Hélène Machinal (éd.), Rennes, PUR, 2014, p. 99-118.

16. Les séries peuvent se démarquer du cinéma contemporain qui produit plutôt des fables dystopiques autour de l'amour impossible entre humain et machine : Her de Spike Jonze (2013) ou Ex Machina d'Alex Garland (2014).

17. On peut cependant rappeler que cette dimension était présente dans Star Trek, l'une des séries pionnières sur les déclinaisons d'une altérité à l'humain.

18. On se souviendra évidemment sur ce point de l'hypotexte cinématographique des "séries » cinématographiques des Blade Runner, Terminator et Matrix.

19. Parmi de très nombreux exemples : https://www.phonandroid.com/intelligence-artificielledans-le-smartphone-concretement-a-quoi-ca-sert.html.

20. Voir les livres du « futuriste » Raymond Kurzweil, et notamment The Singularity is Near: When Humans Transcend Biology, New York, Viking, 2005. Voir aussi le film Marjorie Prime (Michael Almereyda, 2017).

21. Gaïd Girard a récemment, elle aussi, analysé les interactions entre hommes et robots sous le prisme de « la vallée de l'étrange. Voir Gaïd Girard, «Dans "la vallée de l'étrange" : des robots et des hommes, dans trois films de SF américains des années soixante-dix (THX 1138, Westworld, Futureworld) », in Mutations II : homme/machine, op. cit. p. 151-163.

22. Sur la culture de l'écran et ses traits définitoires, voir Bertrand Gervais, «Est-ce maintenant ?/Is it now ? Réflexions sur le contemporain et la culture de l'écran ", in Soif de réalité , Bertrand Gervais, Samuel Archibald, Sylvain David, Joanne Lalonde, Vincent Lavoie, Sylvano Santini (éd.), Montréal, Nota Bene, 2018, p. 17-46.

23. Gilles Lipovetsky, Jean Serroy, L'écran global: culture-médias et cinéma à l'âge hypermoderne. Paris, Éd. Le Seuil, coll. La couleur des idées, 2007.

24. Marcello Vitali-Rosati, Corps et virtuel, Paris, L'Harmattan, 2010.

25. L'article étend la réflexion à d'autres épisodes des saisons 3 et 4 - « Nosedive» (S03E01), «Playtest» (S03E02), « Men Against Fire» (S03E05), « Arkangel», (S04E02 ), «Crocodile » (S04E03) et « Black Museum » (S04E06).

\section{AUTEURS}

\section{HÉLÈNE MACHINAL}

Hélène Machinal est professeure à l'Université de Bretagne Occidentale à Brest et membre de HCTI (EA 4249). Elle est spécialiste de littérature fantastique, du roman policier et de la fiction spéculative au XIX ${ }^{\mathrm{e}}$ siècle et travaille aussi sur la littérature britannique contemporaine, sur les séries TV, et plus particulièrement les fictions policières, fantastiques et de SF. Ses publications récentes incluent : Le Savant fou, Presses Universitaires de Rennes, 2013 ; PostHumain(s). Frontières, évolutions, hybridités (avec Elaine Després), PUR, 2014 ; Mutations I : corps posthumains (avec JeanFrançois Chassay), Otrante n 37, Paris, Kimé, 2015 ; Sherlock (avec Gilles Menegaldo et Jean-Pierre Naugrette), PUR, 2016 ; Signatures du monstre (avec Jean-François Chassay et M. MarracheGouraud) PUR, 2017 ; L'imaginaire en séries I (avec Elaine Després), Otrante n 42, Paris, Kimé, 2017; et Hybridités posthumaines - cyborgs, mutants, hackers (avec Isabelle Boof-Vermesse et Mathieu Freyheit), Paris, Orizons, 2018. 
Hélène Machinal is Full Professor at the University of Bretagne Occidentale, Brest (EA 4249, HCTI). She is a scholar in English Studies, more specifically in British literature, from the $19^{\text {th }}$ century to the contemporary period. She focuses on the gothic, science fiction and detective fiction and her recent research is on TV series. Her recent publications include Le Savant fou (Presses Universitaires de Rennes, 2013); PostHumain(s). Frontières, évolutions, hybridités (with Elaine Després), PUR, 2014; Otrante No. 37: Mutations I : Corps posthumains (with Jean-François Chassay), Kimé, 2015; Sherlock (with Gilles Menegaldo and Jean-Pierre Naugrette) PUR, 2016; Signatures du monstre (with Jean-François Chassay and M. Marrache-Gouraud) PUR, 2017; L'imaginaire en séries I (with Elaine Després), Otrante No. 42, Paris, Kimé, 2017, and Hybridités posthumaines - cyborgs, mutants, hackers (with Isabelle Boof-Vermesse and Mathieu Freyheit), Paris, Orizons, 2018.

\section{MONICA MICHLIN}

Monica Michlin est professeure d'études américaines contemporaines à l'Université Paul Valéry Montpellier 3 (Univ Paul Valery Montpellier 3, EMMA EA 741, 34000 Montpellier). Sa recherche et ses publications portent sur la littérature africaine américaine et LGBTQ, le cinéma et les séries télévisées. Elle s'intéresse notamment aux représentations de minorités ethniques et sexuelles et aux représentations du pouvoir et de la guerre à l'écran. Sur la question du posthumain, elle a notamment édité Philosopher avec Battlestar Galactica, TV/Series ${ }^{\circ} 11$ (juillet 2017).

Monica Michlin is Full Professor of Contemporary American Studies. Her research and publications are in African-American literature, LGBTQ literature, American film and TV series. She focuses on representations of ethnic minorities and LGBTQ characters, as well as on representations of political power and of war on screen. On the specific issue of the posthuman, she is the editor of TV/Series No. 11, on Battlestar Galactica (July 2017). 